\title{
Espectro Vibracional no Infravermelho Próximo dos Polímeros Poliestireno, Poli(Metacrilato de Metila) e Policarbonato
}

\author{
Sérgio C. de Araújo, Yoshio Kawano \\ Instituto de Química, USP
}

Resumo: Os espectros no infravermelho próximo (NIR) dos polímeros amorfos poliestireno (PS), poli(metacrilato de metila) (PMMA) e policarbonato (PC) foram estudados. A tentativa de atribuição das bandas harmônicas e de combinação dos modos vibracionais do PS, PMMA e PC foi realizada baseando-se na teoria de modos locais e pelo uso do método matemático de ajuste de curvas. A correção de anarmonicidade e freqüência mecânica foi determinada em um gráfico de Birge-Sponer. Uma correção de anarmonicidade de 57 e $58 \mathrm{~cm}^{-1}$ foi obtida para os modos de estiramento dos grupos $\mathrm{CH}_{2}$ e $\mathrm{CH}$ do PS; 59 e $9 \mathrm{~cm}^{-1}$ para os modos de estiramento dos grupos $\mathrm{CH}_{3}$ e $\mathrm{CO}$ do PMMA e 53, 59 e $10 \mathrm{~cm}^{-1}$ para os modos de estiramento dos grupos $\mathrm{CH}, \mathrm{CH}_{3}$ e $\mathrm{CO}$ do $\mathrm{PC}$, respectivamente.

Palavras-chave: Infravermelho próximo, poliestireno, poli(metacrilato de metila), policarbonato.

\section{Near-Infrared Spectra of Polystyrene, Poly(Methyl Methacrylate) and Polycarbonate}

\begin{abstract}
The near-infrared (NIR) spectra of the amorphous polymers polystyrene (PS), poly(methyl methacrylate) (PMMA), and polycarbonate (PC) have been studied. The tentative assignment of the overtone and combination frequencies is made using the curve fitting calculations and the local mode theory. Anharmonicity correction and mechanical frequency were determined from a Birge-Sponer plot. A tentative assignment of stretch overtone frequency of $\mathrm{CH}_{2}$ and $\mathrm{CH}$ functional groups of $\mathrm{PS} ; \mathrm{CH}_{3}$ and $\mathrm{CO}$ functional groups of PMMA and $\mathrm{CH}, \mathrm{CH}_{3}$ and $\mathrm{CO}$ functional groups of $\mathrm{PC}$ has been made. An anharmonicity correction of 57 and $58 \mathrm{~cm}^{-1}$ was obtained for $\mathrm{CH}_{2}$ and $\mathrm{CH}$ stretch modes of PS; 59 and $9 \mathrm{~cm}^{-1}$ for $\mathrm{CH}_{3}$ and $\mathrm{CO}$ stretch modes of PMMA and 53, 59 and $10 \mathrm{~cm}^{-1}$ for $\mathrm{CH}, \mathrm{CH}_{3}$ and $\mathrm{CO}$ stretch modes of PC, respectively.
\end{abstract}

Keyword: Near-infrared, polystyrene, poly(methyl methacrylate), polycarbonate.

\section{Introdução}

Em contraste com o número de investigações científicas que visam obter aplicações práticas da espectroscopia NIR (12000 a $4000 \mathrm{~cm}^{-1}$ ) para a análise de propriedades físicas, químicas, controle de processo e de qualidade de sistemas poliméricos ${ }^{[1-6]}$, os estudos que fornecem a base para a correlação entre o espectro NIR e uma propriedade desejada têm sido relegados para segundo plano e não têm sido investigados em uma extensão igual a da região do espectro infravermelho médio ${ }^{[7,8]}$.
Esse fato se deve em parte às dificuldades experimentais, em parte à complexidade de interpretação do espectro NIR e, muito provavelmente, também devido ao uso da espectroscopia NIR em materiais poliméricos não se encontrar em um estágio avançado. Devido a avanços instrumentais e matemáticos ocorridos principalmente nos anos 80 , entretanto, algumas atribuições baseadas na teoria de modos locais do espectro NIR embora empíricas, foram realizadas para alguns polímeros estudados em solução ${ }^{[9,10]}$.

A teoria de modos normais, utilizada pelos espectroscopistas para analisar espectros vibracionais,

Autor para correspondência: Yoshio Kawano, Universidade de São Paulo, Instituto de Química, C.P. 26077, CEP: 05513-970, São Paulo, SP. E-mail: ykawano@iq.usp.br 
considera que, nas moléculas poliatômicas, a vibração dos átomos em determinadas freqüências é composta de modos vibracionais de deformação angular, estiramento simétrico e assimétrico, e outros tipos de modos vibracionais que são considerados movimentos naturais de vibração das moléculas poliatômicas e que estes movimentos vibracionais seguem uma lei de força harmônica. A aproximação harmônica é válida para amplitudes vibracionais infinitesimais em torno da posição de equilíbrio. Para amplitudes finitas (movimentos vibracionais anarmônicos), entretanto, a teoria de modos normais e coordenadas normais associadas encontram dificuldades para descrever as transições vibracionais de alta energia.

Considerando que apenas alguns tipos de movimentos vibracionais (notadamente os estiramentos $\mathrm{CH}, \mathrm{OH}, \mathrm{NH}$ e $\mathrm{SH}$ ) costumam apresentar bandas na região NIR, provenientes de transições harmônicas ou de combinações, foi introduzido o conceito de modo local, em 1975, para analisar os espectros no NIR. Os espectros harmônicos na região NIR podem ser considerados em termos de modo local, isto é, modo associado ao estiramento de uma ligação química individual, aquela que apresenta maior anarmonicidade comparada a outros modos vibracionais da molécula. Um exemplo típico em polímero é o grupo - $\mathrm{CH}_{2^{-}}$, que apresenta o modo de estiramento simétrico e assimétrico. Sabe-se da teoria que a anarmonicidade se torna mais importante com o aumento da energia vibracional, assim o acoplamento entre os dois modos normais se fortalecerá com o aumento da energia vibracional, e a molécula vibrará mais e mais com o perfil vibracional de modo local. A descrição de modo local, portanto, se tornará mais apropriada com o aumento da energia vibracional.

O entendimento da correlação espectro NIR-estrutura, por sua vez, adquiriu ainda maior importância, no momento que polímeros amorfos com elevada qualidade óptica começaram a ser desenvolvidos e utilizados nas aplicações de fotônica ${ }^{[11,12]}$, e posteriormente, lasers de diodos, com comprimentos de onda situados na região NIR, foram desenvolvidos para a comunicação e transmissão de dados ${ }^{[13-15]}$.

Um profundo entendimento do espectro NIR de materiais poliméricos é importante, tanto para uma melhor aplicação da técnica na análise de propriedades químicas e físicas dos polímeros em geral, como para a aplicação dos polímeros PS, PMMA e PC em artefatos como fibras ópticas, onde poderá haver necessidade de se adequar algumas modificações na estrutura molecular do polímero (deuteração, fluoração etc) a fim de remover a atenuação (perda óptica) nas regiões NIR, de interesse.

Este trabalho tem como objetivos estudar a complexidade intrínseca do espectro NIR e realizar uma tentativa de atribuição dos espectros NIR dos polímeros PS, PMMA e PC no estado sólido, por meio da utilização de técnicas matemáticas de ajuste de curva (onde se usa um programa, que decompõe o perfil da banda sobreposta em um conjunto de bandas individuais, cujo somatório das áreas é aproximadamente igual à área da banda sobreposta) e deconvolução espectral (um procedimento matemático que aumenta a resolução de um espectro, por exemplo, de duas bandas próximas, por meio do estreitamento da largura de banda na semi-altura, alterando portanto a forma e a área da banda em relação ao da banda original), de dados disponíveis na literatura, e do método de Birge - Sponer ${ }^{[16]}$. O método de Birge-Sponer consiste em construir um gráfico de $\Delta \mathrm{E} / \mathrm{v}$ em função de $\mathrm{v}$, de acordo com a equação 1 , que fornece uma reta, em que a inclinação corresponde ao valor da correção de anarmonicidade e o coeficiente linear, ao valor da freqüência mecânica.

$$
\Delta \mathrm{E}=\left(\omega_{\mathrm{e}}-\omega_{\mathrm{e}} \chi_{\mathrm{e}}\right) \mathrm{v}-\omega_{\mathrm{e}} \chi_{\mathrm{e}} \mathrm{v}^{2}
$$

Onde $\Delta \mathrm{E}$ é a energia de transição vibracional, $\omega_{\mathrm{e}}$ a freqüência mecânica e $\omega_{\mathrm{e}} \chi_{\mathrm{e}}$ é a correção de anarmonicidade, $\chi_{\mathrm{e}}$ é a constante de anarmonicidade e v é o número quântico vibracional. Este trabalho tem, também, como objetivo verificar a validade da teoria de modo local na interpretação dos espectros.

\section{Experimental e Preparação para Análise NIR}

\section{Materiais}

As placas de PS, PMMA e PC, utilizadas para a obtenção dos espectros NIR, foram preparadas como descrito a seguir: Filmes ( 0,1 a $0,9 \mathrm{~mm}$ de espessura) de PS e PMMA foram preparados pela técnica de vazamento das respectivas soluções. Aproximadamente $3 \mathrm{~g}$ desses materiais foram dissolvidos nos seguintes solventes, sob agitação magnética: PS em tetra-hidrofurano e PMMA em triclorometano. Os solventes (MERCK) foram utilizados como obtidos, sendo que $30 \mathrm{~mL}$ dos mesmos foram suficientes para 
Tabela 1. Configuração do espectrômetro FTIR utilizada para obtenção de espectros no NIR.

\begin{tabular}{cccc}
\hline $\begin{array}{c}\text { Número } \\
\text { de onda } \\
\left(\mathbf{c m}^{-1}\right)\end{array}$ & Fonte & $\begin{array}{c}\text { Divisor } \\
\text { de feixe }\end{array}$ & Detetor \\
\hline 3500 a 5500 & Globar & $\mathrm{KBr}$ & DTGS \\
4500 a 9000 & $\begin{array}{c}\text { Quartzo/ } \\
\text { Halogênio } \\
\text { Quartzo/ }\end{array}$ & Visível & MCT \\
8500 a 12500 & $\begin{array}{c}\text { Visível } \\
\text { Halogênio }\end{array}$ & $\mathrm{Si}$ \\
\hline
\end{tabular}

se obter uma boa dissolução em todos os casos. Os filmes com espessuras aproximadas de $1 \mathrm{~mm}$ de PC, foram obtidos por injeção, em uma máquina injetora da NETSTAL. Não foi possível obter filmes de PC com a espessura acima de $0,1 \mathrm{~mm}$ por vazamento da solução. Filmes mais espessos de PS, PMMA e PC também foram utilizados, superpondo-se vários filmes.

\section{Instrumentação}

Os espectros no NIR dos polímeros PS, PMMA e PC foram obtidos em um espectrômetro FTIR, da Bomem, modelo DA3, utilizando a configuração apresentada na Tabela 1 .

Os espectros NIR do PS, PMMA e do PC foram obtidos com uma resolução de $4 \mathrm{~cm}^{-1}$, todos com apodização Hamming.

\section{Resultados e Discussão}

\section{Poliestireno}

A estrutura do mero do poliestireno sugere que para este polímero existem três tipos de ligações $\mathrm{CH}$ não equivalentes: $\mathrm{CH}_{2}, \mathrm{CH}$ e $\mathrm{CH}$ aromático.

\section{Mero do poliestireno}

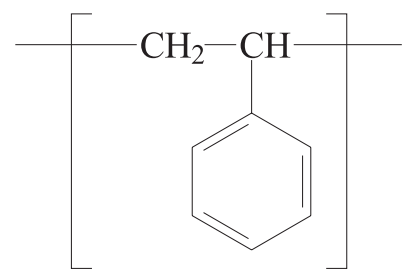

O espectro no infravermelho médio (MIR) do PS e o assinalamento das bandas observadas no espectro estão disponíveis na literatura ${ }^{[17,18]}$.

Uma análise do espectro NIR, apresentado na Figura 1, revela que na região entre 4500 e $3600 \mathrm{~cm}^{-1}$ são observadas uma série de bandas que devem ser originadas de combinações e/ou harmônicas de deformação angular das vibrações fundamentais. O PS apresenta uma absorção muito fraca na região próxima a 9500 e $7500 \mathrm{~cm}^{-1}$. Outra característica do espectro NIR do PS, que tem sido observada e descrita na literatura ${ }^{[3,5]}$ para outros polímeros contendo anel aromático, é a nítida separação entre as absorções aromáticas e alifáticas nas regiões de harmônicas superiores $(\Delta \mathrm{v} \geq 3)$ como pode ser observado entre 9000 a 8000 e 11500 a $10500 \mathrm{~cm}^{-1}$, respectivamente nas Figuras 1A e 1B. Pode ser observado também na Figura 1A, que o PS apresenta um espectro complexo na região entre 6200 e $5400 \mathrm{~cm}^{-1}$, constituído de bandas que podem ser atribuídas, ainda sob uma aproximação de modos normais, a harmônicas ou combinações dos modos vibracionais de estiramento simétrico e assimétrico dos grupos funcionais do polímero.

Na região fundamental, adicionalmente, as absorções devidas ao estiramento $\mathrm{CH}$ do grupo alifático e do grupo aromático ocorrem na mesma região, dificultando ainda mais a atribuição das bandas do espectro NIR. Considera-se dessa forma, que o espectro

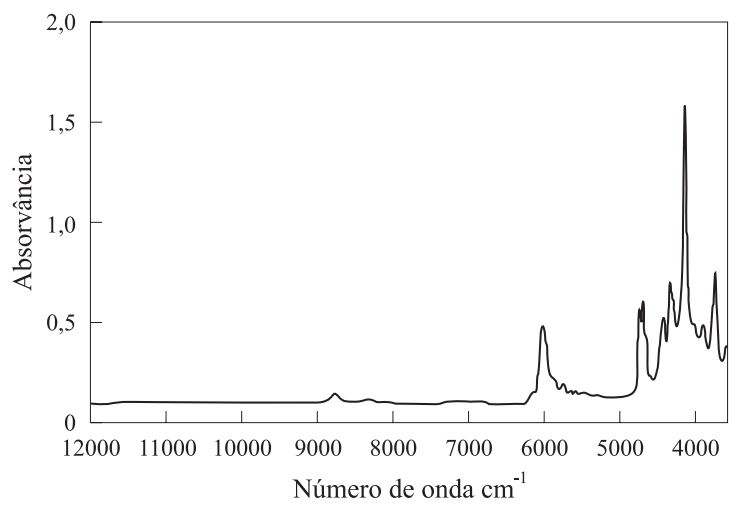

Figura 1A. Espectro no NIR do PS. Espessura do filme 0,9 mm.

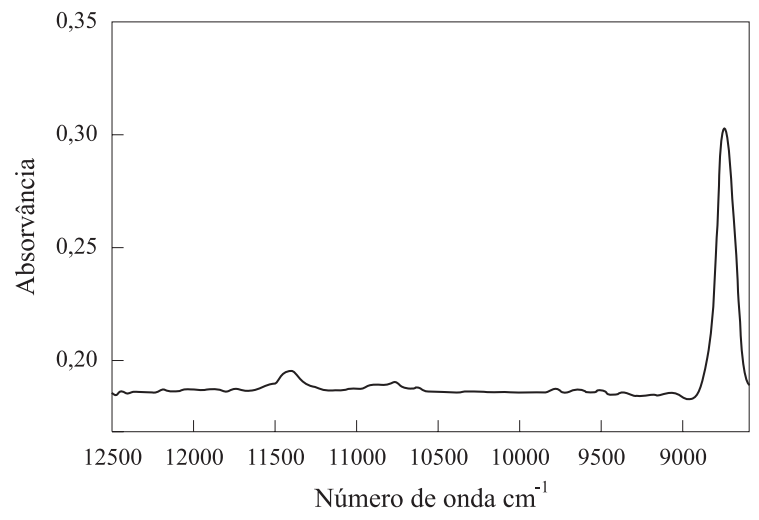

Figura 1B. Espectro NIR do PS. Espessura do filme 4,5 mm. 


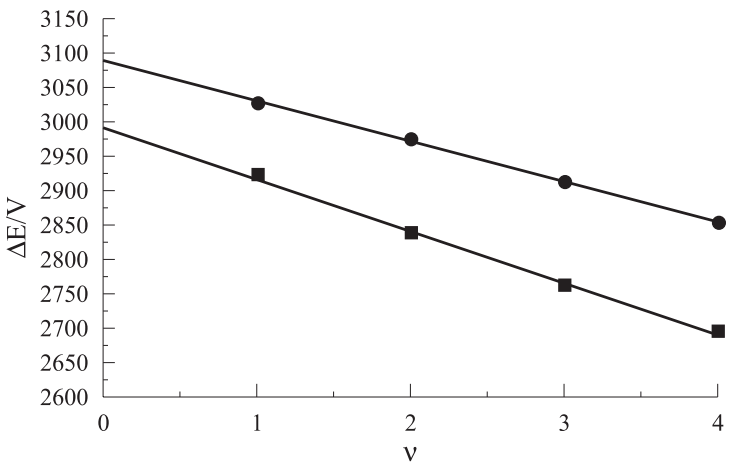

Figura 2. Gráfico de Birge-Sponer. Os valores utilizados são descritos no texto. n Estiramento $\mathrm{CH}\left(\mathrm{de} \mathrm{CH}_{2}\right)$; 1 Estiramento $\mathrm{CH}$ (do anel)

Tabela 2. Correções de anarmonicidade e freqüências mecânicas calculadas a partir da Figura 2

\begin{tabular}{ccc}
\hline Grupo & $\begin{array}{c}\omega_{\mathrm{e}} \chi_{\mathrm{e}} \\
\left(\mathbf{c m}^{-1}\right)\end{array}$ & $\begin{array}{c}\boldsymbol{\omega}_{\mathrm{e}} \\
\left(\mathbf{c m}^{-1}\right)\end{array}$ \\
\hline $\mathrm{CH}_{2}$ & $74( \pm 4)$ & 2991 \\
$\mathrm{CH}$ & $58( \pm 2)$ & 3087
\end{tabular}

Onde: $\omega_{\mathrm{e}} \chi_{\mathrm{e}}=$ correção de anarmonicidade e $\omega_{\mathrm{e}}=$ freqüência mecânica. $\mathrm{O}$ valor entre parêntesis é o desvio padrão

NIR do PS é originado pelas progressões de dois osciladores anarmônicos $\mathrm{CH}$ e $\mathrm{CH}_{2}$.

Considerando as bandas mais fortes na região fundamental de estiramento e os máximos das bandas harmônicas atribuídas às absorções de estiramento dos grupos $\mathrm{CH}_{2}$ e $\mathrm{CH}$ aromático as seguintes progressões podem ser identificadas: 2924 (fundamental), 5670 (primeira harmônica), 8286 (segunda harmônica) e 10804 (terceira harmônica) cm $\mathrm{cm}^{-1} \mathrm{e} 3026$ (fundamental), 5955 (primeira harmônica), 8741 (segunda harmônica) e 11415 (terceira harmônica) $\mathrm{cm}^{-1}$, respectivamente. As respectivas correções de anarmonicidade e freqüências mecânicas calculadas a partir das retas de regressão linear apresentadas na Figura 2 são listadas na Tabela 2.

Como pode ser observado na Figura 2, os pontos se ajustam muito bem à reta. Por outro lado, o valor da correção de anarmonicidade obtido para o estiramento $\mathrm{CH}$ do grupo $\mathrm{CH}_{2}$ difere daqueles observados em trabalhos apresentados na literatura para este grupo funcional em polímeros e outras moléculas orgânicas ${ }^{[19]}$.

Em vista desta discrepância, foi realizado um ajuste de curva assumindo uma forma de linha Gaussiana, das bandas da região fundamental e das regiões harmônicas, cujos valores obtidos são listados na Tabela 3.

Vários gráficos de Birge - Sponer foram calcula-

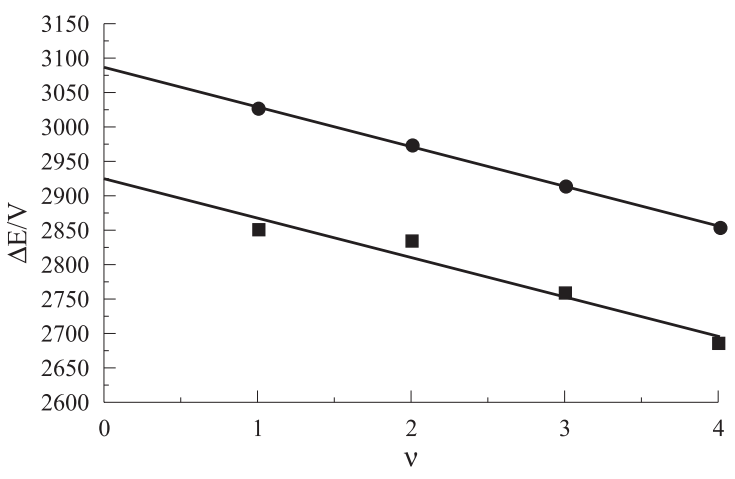

Figura 3. Gráfico de Birge-Sponer. $n$ Grupo $\mathrm{CH}_{2}\left(\mathrm{v}_{\mathrm{s}}\right)$ : $2850 \mathrm{~cm}^{-1}$ (fundamental), $5669 \mathrm{~cm}^{-1}$ (1 $1^{\mathrm{a}}$ harmônica), $8274 \mathrm{~cm}^{-1}$ (2 harmônica), 10745 $\mathrm{cm}^{-1}$ (3 harmônica). 1 Grupo CH (aromático): $3026 \mathrm{~cm}^{-1}$ (fundamental), $5945 \mathrm{~cm}^{-1}$ (1 ${ }^{\mathrm{a}}$ harmônica), $8739 \mathrm{~cm}^{-1}$ (2a harmônica), $11414 \mathrm{~cm}^{-1}$ (3a harmônica).

Tabela 3. Número de onda $\left(\mathrm{cm}^{-1}\right)$ na região fundamental e nas regiões de harmônicas de estiramento, determinadas pelo processo de ajuste de curva.

\begin{tabular}{ll}
\hline Região: Fundamental & $\begin{array}{l}2850,2908,2925,2949,3001, \\
3026,3060,3082\end{array}$ \\
\hline Região: 6500 a 5000 & $\begin{array}{l}5383,5564,5669,5706,5831, \\
5898,5944,5971,6073,6106\end{array}$ \\
\hline Região: 9000 a 8000 & $8179,8274,8319,8506,8739,8855$ \\
\hline Região: 12000 a 10000 & $\begin{array}{l}10461,10575,10733,10855, \\
10917,11369,11413,11522\end{array}$ \\
\hline
\end{tabular}

dos utilizando-se uma combinação de valores apresentados na tabela acima. Da análise desses gráficos e do ajuste de curva, concluiu-se que as progressões mais prováveis são as apresentadas na Figura 3.

Os valores de correção de anarmonicidade e de freqüência mecânica obtidos na Figura 3 são apresentados na Tabela 4 e concordam com os valores da literatura.

Uma tentativa de atribuição do espectro NIR do PS baseada na discussão dos resultados é apresentada na Tabela 5 .

As bandas observadas em 6111 e $6075 \mathrm{~cm}^{-1}$ (Figura 1A) são de difícil atribuição pois podem ser harmônicas das freqüências entre 3103 e $3060 \mathrm{~cm}^{-1}$, respectivamente ou bandas de combinação.

Tabela 4. Correções de anarmonicidade e freqüências mecânicas calculadas na Figura 3.

\begin{tabular}{ccc}
\hline Grupo & $\begin{array}{c}\omega_{\mathrm{e}} \chi_{\mathrm{e}} \\
\left(\mathbf{c m}^{-1}\right)\end{array}$ & $\begin{array}{c}\omega_{\mathrm{e}} \\
\left(\mathbf{c m}^{-1}\right)\end{array}$ \\
\hline $\mathrm{CH}_{2}$ & $57( \pm 10)$ & 2924 \\
$\mathrm{CH}$ & $58( \pm 1)$ & 3085
\end{tabular}

Onde: $\omega_{\mathrm{e}} \chi_{\mathrm{e}}=$ correção de anarmonicidade e $\omega_{\mathrm{e}}=$ frequiência mecânica. $\mathrm{O}$ valor entre parêntesis é o desvio padrão 
Tabela 5. Atribuição provável do espectro NIR do PS.

\begin{tabular}{cccc}
\hline $\begin{array}{c}\text { Número de onda } \\
\left(\mathbf{c m}^{-1}\right)\end{array}$ & Atribuição & $\begin{array}{c}\text { Número de onda } \\
\left(\mathbf{c m}^{-1}\right)\end{array}$ & Atribuição \\
\hline 4327 & $3 \times 1452$ & 8739 & $v(\mathrm{CH}), 2^{\text {a }}$ harmônica \\
5669 & $v\left(\mathrm{CH}_{2}\right), 1^{\text {a }}$ harmônica & 10745 & $v\left(\mathrm{CH}_{2}\right), 3^{\text {a }}$ harmônica \\
5944 & $v(\mathrm{CH}), 1^{\text {a }}$ harmônica & 11414 & $v(\mathrm{CH}), 3^{\text {a }}$ harmônica \\
8274 & $v\left(\mathrm{CH}_{2}\right), 2^{\text {a }}$ harmônica & & \\
\hline
\end{tabular}

Poli(metacrilato de metila)

O espectro MIR do PMMA e a atribuição das freqüências do espectro estão disponíveis na literatu$\mathrm{ra}^{[20,21]}$.

\section{Mero do poli(metacrilato de metila).}<smiles>CCC(C)(C)C(=O)OC</smiles>

Os modos de estiramento $\mathrm{CH}$ e deformação angular dos grupos metileno, metila e metila do grupamento éster se apresentam sobrepostos nas respectivas regiões dificultando o assinalamento dos referidos grupos tanto no MIR como no NIR. O espectro no MIR e NIR do PMMA, na região entre 6000 e $3200 \mathrm{~cm}^{-1}$, é apresentado na Figura 4. A atribuição tentativa das bandas com maior intensidade, utilizando a aproximação empírica de soma e múltiplos das frequiências fundamentais, é apresentado na Tabela 6.

Dentre as várias possibilidades de atribuição para cada uma das freqüências listadas na Tabela 6 , foram consideradas como as mais prováveis, aquelas que apresentaram a menor diferença entre o valor determinado pela soma ou múltiplos da freqüência das bandas na região fundamental e o valor das frequiências apresentadas na Tabela 6 , correspondente às bandas observadas na Figura 4.

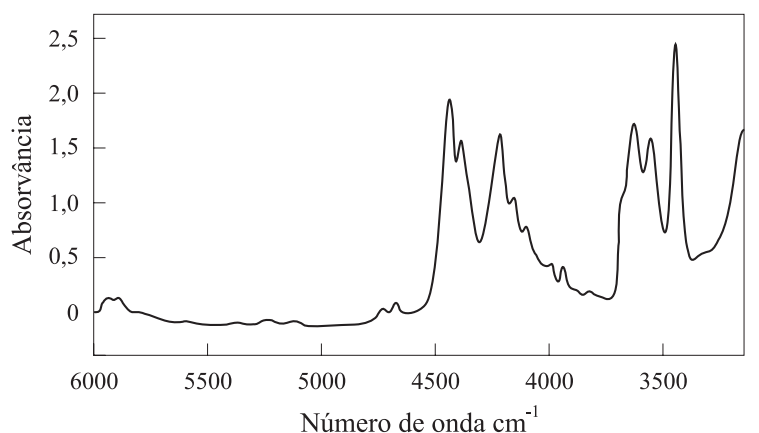

Figura 4. Espectro no MIR e no NIR do PMMA. Espessura do filme $1 \mathrm{~mm}$
A Figura 5A apresenta o espectro NIR do PMMA na região entre 12000 e $4600 \mathrm{~cm}^{-1}$. A Figura 5B apresenta o espectro NIR na região entre 12500 e $8500 \mathrm{~cm}^{-1}$, obtido com uma amostra de $8 \mathrm{~mm}$ de espessura.

Como é difícil diferenciar o conjunto de bandas fundamentais de estiramento simétrico e assimétrico dos grupos $\mathrm{CH}_{2} \mathrm{e} \mathrm{CH}_{3}$ no PMMA, considera-se inicialmente a existência de apenas dois tipos de ligações não equivalentes, ou seja, uma ligação $\mathrm{C}=\mathrm{O}$ e um tipo de ligação $\mathrm{CH}$. Contudo, todos os modos vibracionais de estiramento dos grupos $\mathrm{CH}_{2}$ e $\mathrm{CH}_{3}$ devem estar contribuindo para as bandas NIR ob-

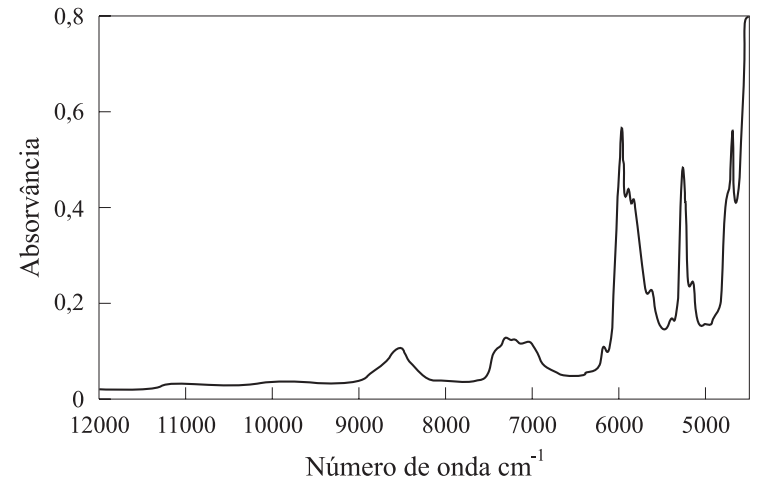

Figura 5A. Espectro NIR do PMMA. Espessura do filme $2 \mathrm{~mm}$.

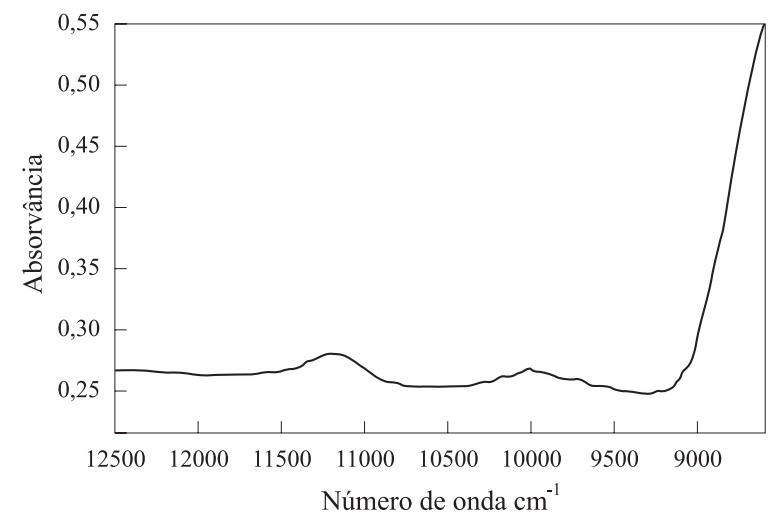

Figura 5B. Espectro NIR do PMMA. Espessura do filme $8 \mathrm{~mm}$. 
Araujo, S. C.; Kawano, Y. - Espectro no infravermelho próximo de polímeros

Tabela 6. Atribuição provável do espectro MIR e NIR do PMMA

\begin{tabular}{|c|c|}
\hline $\begin{array}{l}\text { Número de onda } \\
\qquad\left(\mathrm{cm}^{-1}\right)\end{array}$ & Atribuição \\
\hline 3437 & $2 \times 1730, v(\mathrm{CO})$ \\
\hline 3550 & $3001+554, v_{\mathrm{a}}\left(\mathrm{CH}_{3}\right)+\delta(\mathrm{CCO})$ \\
\hline 3623 & $2841+810, v_{\mathrm{s}}\left(\mathrm{CH}_{3}\right)+\mathrm{v}(\mathrm{COC})$ \\
\hline 3941 & $4 \times 989, \rho\left(\mathrm{CH}_{3}\right)$ de $\mathrm{OCH}_{3}$ \\
\hline 4099 & $3 \times 1367, \tau\left(\mathrm{CH}_{2}\right)$ \\
\hline 4154 & $3 \times 1387, \delta\left(\mathrm{CH}_{3}\right)$ de $\mathrm{CCH}_{3}$ \\
\hline 4213 & 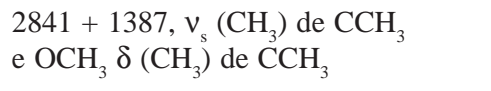 \\
\hline 4387 & $\begin{array}{l}3001+1387, v_{\mathrm{a}}\left(\mathrm{CH}_{3}\right) \text { de } \mathrm{CCH}_{3} \\
\mathrm{e} \mathrm{OCH}_{3}+\delta\left(\mathrm{CH}_{3}\right) \mathrm{de} \mathrm{CCH}_{3}\end{array}$ \\
\hline 4436 & $3001+1436, v_{a}\left(\mathrm{CH}_{3}\right)$ de $\mathrm{CCH}_{3}$ e $\mathrm{OCH}_{3}$ \\
\hline
\end{tabular}

Onde: $v=$ estiramento, $v_{\mathrm{a}}=$ estiramento assimétrico, $v_{\mathrm{s}}=$ estiramento simétrico, $\rho=$ "rocking", $\tau=$ "twisting" e $\delta=$ deformação angular.

servadas nos espectros apresentados nas Figuras 5A e $5 \mathrm{~B}$.

A deconvolução e ajuste de curva, assumindo a forma de linha Gaussiana, do espectro no NIR entre 12000 e $6500 \mathrm{~cm}^{-1}$, não evidenciou, ao contrário do que era esperado, frequiências que pudessem ser atribuídas imediatamente aos diferentes modos de estiramento $\mathrm{CH}$, sugerindo que para esta região, o espectro NIR do PMMA é melhor explicado pela teoria de modo local.

A Tabela 7 apresenta as freqüências observadas na região fundamental do estiramento $\mathrm{CH}$, na região da primeira harmônica $\left(6500\right.$ a $\left.5500 \mathrm{~cm}^{-1}\right)$ e as determinadas no ajuste de curva

Bandas harmônicas de estiramento $\mathrm{C}=\mathrm{O}$ também podem ser identificadas no espectro NIR do PMMA. A primeira harmônica de estiramento $\mathrm{CO}$ foi identificada em $3437 \mathrm{~cm}^{-1}$. A segunda harmônica de CO é atribuída a $5130 \mathrm{~cm}^{-1}$. A terceira harmônica de CO foi atribuída a uma banda em $6768 \mathrm{~cm}^{-1}$, detectada pelo ajuste de curva. A quarta harmônica de $\mathrm{CO}$ cai na região da segunda harmônica de estiramento CH e sua identificação é, portanto, difícil. A sexta

Tabela 7. Freqüências fundamentais e determinadas no ajuste de curva do espectro no NIR

\begin{tabular}{ll}
\hline Região: Fundamental cm-1 & $2841,2929,2951,3001$ \\
\hline Região: 5500 a $6600 \mathrm{~cm}^{-1}$ & $5611,5816,5874,5943$ \\
\hline Região: 8000 a $9000 \mathrm{~cm}^{-1}$ & $8470,8501,8629,8795$ \\
\hline Região: 10500 a $12000 \mathrm{~cm}^{-1}$ & $11049,11159,11296,11510$ \\
\hline
\end{tabular}

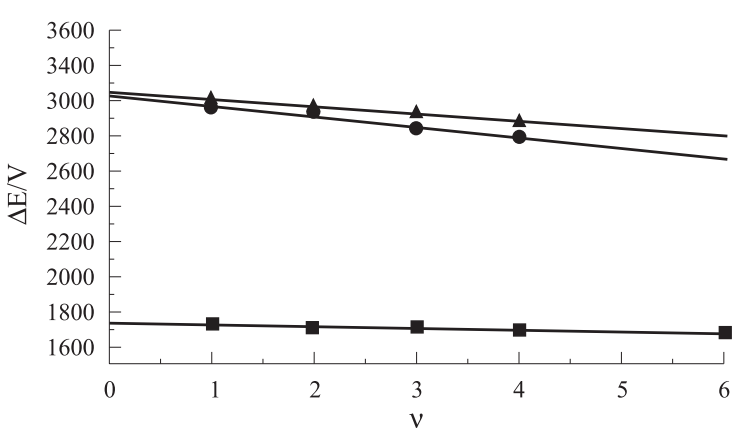

Figura 6. Gráfico de Birge-Sponer. Valores utilizados descrito no texto. n Estiramento CO; 1 Progressão (b); s Progressão (a).

harmônica de $\mathrm{CO}$ foi atribuída a uma banda observada em $10122 \mathrm{~cm}^{-1}$, detectada no ajuste de curva.

A Figura 6 apresenta o gráfico de Birge-Sponer para os estiramentos $\mathrm{CH}$ e $\mathrm{CO}$. A correção de anarmonicidade e freqüência mecânica para o modo de estiramento $\mathrm{CO}$ foi calculada da seguinte progressão: 1730 (fundamental), 3437 ( $1^{\mathrm{a}}$ harmônica), 5130 (2a harmônica), 6768 ( $3^{\text {a }}$ harmônica) e $10122 \mathrm{~cm}^{-1}$ ( $5^{\mathrm{a}}$ harmônica). Das várias possibilidades investigadas para a progressão de estiramento $\mathrm{CH}$, as mais prováveis são: a) $3001,5943,8795,11510 \mathrm{~cm}^{-1}$ e b) 2951 , $5874,8501,11159 \mathrm{~cm}^{-1}$.

A progressão (b) foi a que apresentou valores de correção de anarmonicidade e de frequiência mecânica próximos aos observados na literatura para o estiramento $\mathrm{CH}$. Os valores das correções de anarmonicidade e de frequiência mecânica obtidos a partir dessas retas são apresentados na Tabela 8.

Uma possível atribuição do espectro no NIR, de acordo com as considerações mencionadas, é a apresentada na Tabela 9. As bandas observadas entre 7600 e 6700 e entre 10500 e $8500 \mathrm{~cm}^{-1}$ são provavelmente originadas da combinação de uma banda harmônica mais um múltiplo de uma banda fundamental de deformação angular. As bandas observadas em $7308 \mathrm{~cm}^{-1}$ e em $9963 \mathrm{~cm}^{-1}$ podem ser atribuídas à combinação $(5874+1436)$ e $(8501+1436)$ respectivamente.

Tabela 8. Correção de anarmonicidade e freqüências mecânicas determinadas a partir da Figura 9.

\begin{tabular}{ccc}
\hline Grupo & $\begin{array}{c}\omega_{\mathrm{e}} \chi_{\mathrm{e}} \\
\left(\mathbf{c m}^{-1}\right)\end{array}$ & $\begin{array}{c}\omega_{\mathrm{e}} \\
\left(\mathbf{c m}^{-1}\right)\end{array}$ \\
\hline Progressão (b): & $59( \pm 11)$ & 3024 \\
Progressão (a): & $41( \pm 4)$ & 3048 \\
CO & $9( \pm 2)$ & 1736 \\
\hline
\end{tabular}

Onde: $\omega_{\mathrm{e}} \chi_{\mathrm{e}}=$ correção de anarmonicidade e $\omega_{\mathrm{e}}=$ freqüência mecânica. $O$ valor entre parêntesis é o desvio padrão 
Tabela 9. Atribuição provável do espectro NIR do PMMA

\begin{tabular}{|c|c|}
\hline $\begin{array}{c}\text { Número } \\
\text { de onda } \\
\left(\mathrm{cm}^{-1}\right)\end{array}$ & Atribuição \\
\hline 3437 & $1^{a}$ harmônica, $v(\mathrm{C}=\mathrm{O})$ \\
\hline 5130 & $2^{\text {a }}$ harmônica, $v(\mathrm{C}=\mathrm{O})$ \\
\hline 5611 & $1^{\mathrm{a}}$ harmônica, da frequiência $2841 \mathrm{~cm}^{-1}(\mathrm{v} \mathrm{CH})$ \\
\hline 5816 & $1^{a}$ harmônica, da frequiência $2929 \mathrm{~cm}^{-1}(\mathrm{v} \mathrm{CH})$ \\
\hline 5874 & $1^{\text {a }}$ harmônica, da frequiência $2951 \mathrm{~cm}^{-1}(v \mathrm{CH})$ \\
\hline 5943 & $1^{\text {a }}$ harmônica, da frequiência $3001 \mathrm{~cm}^{-1}(v \mathrm{CH})$ \\
\hline 6768 & $3^{\mathrm{a}}$ harmônica, $v(\mathrm{C}=\mathrm{O})$ \\
\hline 8501 & $2^{a}$ harmônica, da frequiência $2951 \mathrm{~cm}^{-1}(\mathrm{v} \mathrm{CH})$ \\
\hline 8795 & $2^{a}$ harmônica, da frequiência $3001 \mathrm{~cm}^{-1}(v \mathrm{CH})$ \\
\hline 10122 & $5^{\text {a }}$ harmônica, $v(\mathrm{C}=\mathrm{O})$ \\
\hline 11159 & $3^{a}$ harmônica, da frequiência $2951 \mathrm{~cm}^{-1}(\mathrm{v} \mathrm{CH})$ \\
\hline 11510 & $3^{\mathrm{a}}$ harmônica, da frequiência $3001 \mathrm{~cm}^{-1}(\mathrm{v} \mathrm{CH})$ \\
\hline
\end{tabular}

\section{Policarbonato}

Mero do policarbonato

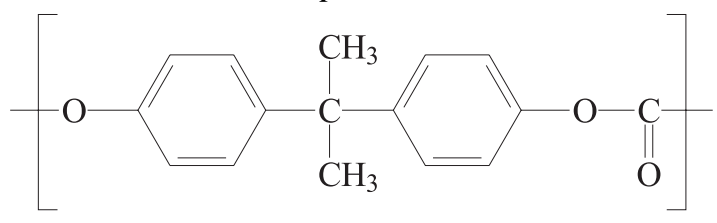

O espectro MIR do PC está disponível na literatura, e o assinalamento das bandas foi realizado com base nas atribuições espectrais dos respectivos grupos constituintes.

O espectro NIR do PC é apresentado na Figura 7A. Na Figura 7B, é apresentado o espectro do PC na região entre 12500 e $8500 \mathrm{~cm}^{-1}$, obtido de um filme com $7 \mathrm{~mm}$ de espessura.

Pode-se dizer, pela fórmula química do mero, que o espectro NIR do PC será composto dos movimentos vibracionais de três grupos não equivalentes, $\mathrm{C}=\mathrm{O}, \mathrm{CH}_{3}$ e $\mathrm{CH}$ aromático. Como esses grupos

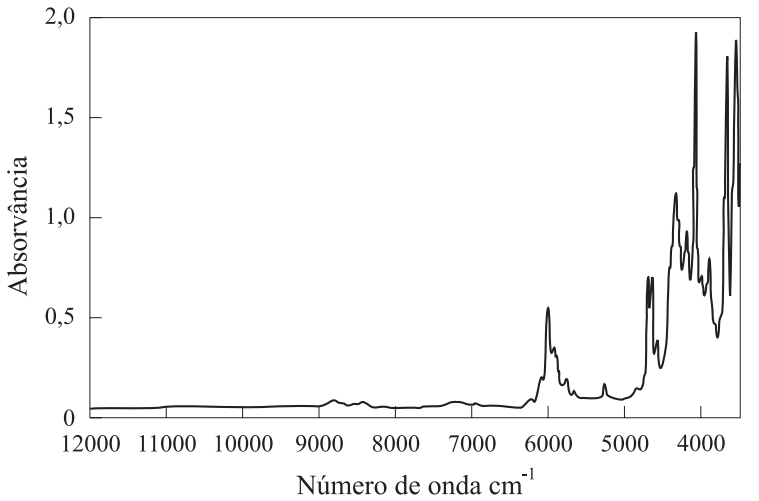

Figura 7A. Espectro NIR do PC. Espessura do filme $1 \mathrm{~mm}$.

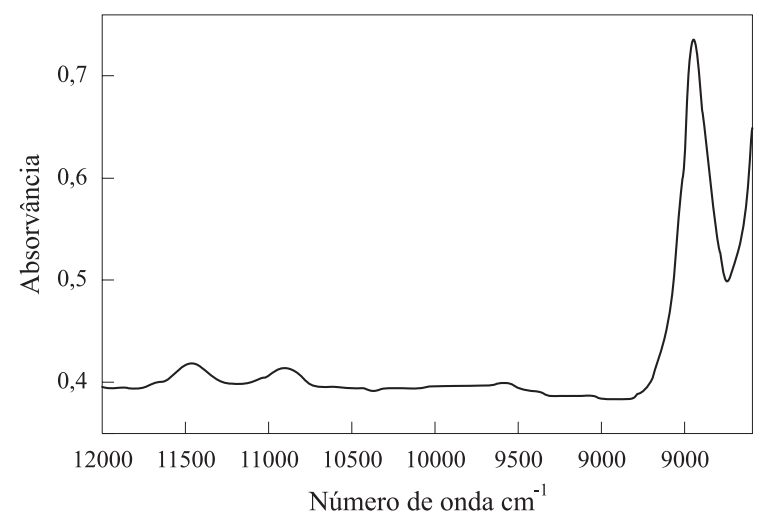

Figura 7B. Espectro NIR do PC. Espessura do filme $7 \mathrm{~mm}$.

absorvem em números de onda bem separados na região fundamental, é fácil identificar no espectro NIR, principalmente acima de $6000 \mathrm{~cm}^{-1}$, absorções que podem ser atribuídas aos grupos aromáticos e alifáticos, onde se espera detectar as respectivas bandas harmônicas.

Como ocorreu para o PS e para o PMMA, na região onde aparecem as primeiras harmônicas de estiramento $\left(6000\right.$ e $\left.5500 \mathrm{~cm}^{-1}\right)$, ainda podem ser vistas bandas resolvidas, com o perfil espectral sugerido pelo acoplamento de vários modos normais. Acima de $6000 \mathrm{~cm}^{-1}$, o perfil espectral é melhor explicado pela aproximação de modos locais.

Tabela 10. Atribuição provável do espectro MIR e NIR na região entre 5000 e $3600 \mathrm{~cm}^{-1}$

\begin{tabular}{cccc}
\hline $\begin{array}{c}\text { Número de onda } \\
\left(\mathbf{c m}^{-1}\right)\end{array}$ & Atribuição & $\begin{array}{c}\text { Número de onda } \\
\left(\mathbf{c m}^{-1}\right)\end{array}$ & Atribuição \\
\hline 3556 & $2 \times 1778$ & 4416 & $2908+1508$ \\
3674 & $2908+766$ & 4576 & \\
3889 & $2873+1016$ & 4642 & $3040+1602$ \\
4095 & $3 \times 1365$ & 4686 & $2908+1778$ \\
4339 & $2873+1466$ & 4844 & \\
\hline
\end{tabular}


Tabela 11. Bandas observadas na região fundamental de estiramento $\mathrm{CH}$, na região da primeira harmônica e no tratamento matemático das bandas observadas nas regiões da segunda e terceira harmônicas.

\begin{tabular}{|c|c|}
\hline Região: Fundamental $\left(\mathrm{cm}^{-1}\right)$ & $\begin{array}{l}2873,2890,2908,2935, \\
2968,3040,3057\end{array}$ \\
\hline Região: 5500 a $6600\left(\mathrm{~cm}^{-1}\right)$ & $5659,5767,5922,5999$ \\
\hline Região: 8000 a $9000\left(\mathrm{~cm}^{-1}\right)$ & $\begin{array}{l}8378,8418,8497,8534 \\
8728,8788,8832,8893\end{array}$ \\
\hline Região: 10500 a $12000\left(\mathrm{~cm}^{-1}\right)$ & $\begin{array}{l}10960,11055,11181,11427, \\
11534,11615,11714\end{array}$ \\
\hline
\end{tabular}

A região compreendida entre 5000 e $3600 \mathrm{~cm}^{-1}$, como mostrado na Figura 7A, apresenta um conjunto de bandas que podem ser atribuídas a harmônicas de modos de deformação angular ou a bandas de combinação de diferentes modos fundamentais. A atribuição dessa região espectral é apresentada na Tabela 10.

A atribuição feita na Tabela 10 foi realizada pela aproximação de soma e múltiplos das freqüências fundamentais, sendo escolhidos como mais prováveis os resultados que apresentaram a menor diferença entre os valores das frequiências calculadas e os apresentados na Tabela 10 , pois é conhecido que a anarmonicidade é pequena para modos de deformação angular (tipicamente $\sim 10 \mathrm{~cm}^{-1}$ ).

Bandas observadas pelo tratamento de ajuste de curva, assumindo uma forma de linha Gaussiana, nas regiões de 9000 a $8350 \mathrm{~cm}^{-1}$ e de 11800 a $10800 \mathrm{~cm}^{-1}$, respectivamente, são listadas na Tabela 11 , juntamente com as bandas dos modos de estiramento observadas na região fundamental.

Da análise de várias possibilidades de atribuição, as seguintes progressões de estiramento para os grupos $\mathrm{C}=\mathrm{O}, \mathrm{CH}_{3}$ e $\mathrm{CH}$ foram identificadas como as mais prováveis, respectivamente: $1778 \mathrm{~cm}^{-1}$ (fundamental), $3534 \mathrm{~cm}^{-1}$ (1 $1^{\text {a }}$ harmônica), $5264 \mathrm{~cm}^{-1}$ ( $2^{\text {a }}$ harmônica), $6998 \mathrm{~cm}^{-1}$ (3 harmônica); $2935 \mathrm{~cm}^{-1}$ (fundamental), $5767 \mathrm{~cm}^{-1}$ (1 $1^{\mathrm{a}}$ harmônica), $8418 \mathrm{~cm}^{-1}$ ( $2^{\mathrm{a}}$ harmônica),

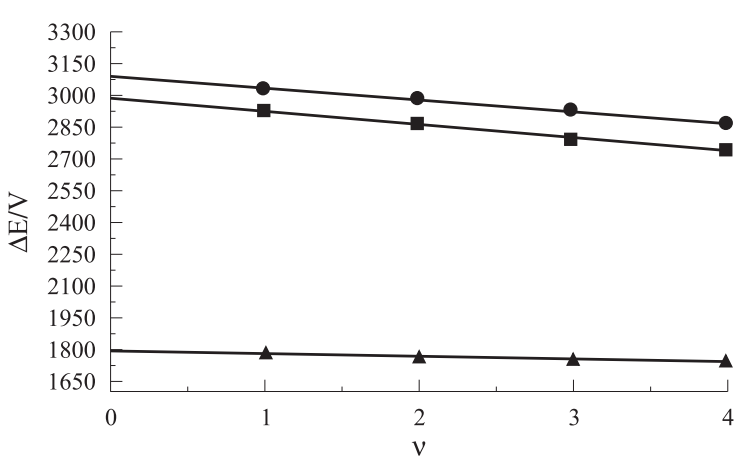

Figura 8. Gráfico de Birge-Sponer. Valores utilizados são apresentados no texto. s Estiramento $\mathrm{CO}$; $\mathrm{n}$ Estiramento $\mathrm{CH}_{3}$; 1 Estiramento $\mathrm{CH}$.

Tabela 12. Correção de anarmonicidade e freqüências mecânicas obtidas a partir da Figura 8, para os grupos $\mathrm{CO}, \mathrm{CH}_{3}$ e $\mathrm{CH}$.

\begin{tabular}{ccc}
\hline Grupo & $\begin{array}{c}\omega_{\mathrm{e}} \chi_{\mathrm{e}} \\
\left(\mathbf{c m}^{-1}\right)\end{array}$ & $\begin{array}{c}\omega_{\mathrm{e}} \\
\left(\mathbf{c m}^{-1}\right)\end{array}$ \\
\hline $\mathrm{CO}$ & $10( \pm 1)$ & 1787 \\
$\mathrm{CH}_{3}$ & $59( \pm 4)$ & 2994 \\
$\mathrm{CH}$ & $53( \pm 3)$ & 3098
\end{tabular}

Onde: $\omega_{\mathrm{e}} \chi_{\mathrm{e}}=$ correção de anarmonicidade e $\omega_{\mathrm{e}}=$ freqüência mecânica. $\mathrm{O}$ valor entre parêntesis é o desvio padrão

$11055 \mathrm{~cm}^{-1}$ ( $3^{\text {a }}$ harmônica) e $3040 \mathrm{~cm}^{-1}$ (fundamental), $5999 \mathrm{~cm}^{-1}$ (1 $1^{\text {a }}$ harmônica), $8832 \mathrm{~cm}^{-1}$ (2a harmônica), $11534 \mathrm{~cm}^{-1}$ ( $3^{\text {a }}$ harmônica).

Das retas de regressão linear, obtidas das progressões acima, em um gráfico de Birge-Sponer, apresentado na Figura 8, foram obtidos os valores das correções de anarmonicidade e de freqüência mecânica, apresentados na Tabela 12, para os diferentes grupos.

Os valores apresentados na Tabela 12 para a constante de anarmonicidade e para a freqüência mecânica estão próximos aos valores apresentados na literatura para o estiramento $\mathrm{CH}$ e $\mathrm{CO}$ dos respectivos grupos, investigados para outros polímeros e algumas moléculas orgânicas ${ }^{[17]}$.

Tabela 13. Atribuição provável do espectro NIR do PC

\begin{tabular}{|c|c|c|c|}
\hline $\begin{array}{l}\text { Número de onda } \\
\qquad\left(\mathrm{cm}^{-1}\right)\end{array}$ & Atribuição & $\begin{array}{l}\text { Número de onda } \\
\qquad\left(\mathrm{cm}^{-1}\right)\end{array}$ & Atribuição \\
\hline 3534 & v $(\mathrm{CO}), 1^{\mathrm{a}}$ harmônica & 6998 & v $(\mathrm{CO}), 3^{\mathrm{a}}$ harmônica \\
\hline 5264 & $v(\mathrm{CO}), 2^{\mathrm{a}}$ harmônica & 8418 & $v\left(\mathrm{CH}_{3}\right), 2^{\mathrm{a}}$ harmônica \\
\hline 5659 & $v(\mathrm{CH}), 2 \times 2873$ & 8832 & $v(\mathrm{CH})^{*}, 2^{\mathrm{a}}$ harmônica \\
\hline 5767 & $v\left(\mathrm{CH}_{3}\right), 1^{\mathrm{a}}$ harmônica & 11055 & $v\left(\mathrm{CH}_{3}\right), 3^{\mathrm{a}}$ harmônica \\
\hline 5922 & $v(\mathrm{CH}), 2 \times 2968$ & 11534 & $v(\mathrm{CH})^{*}, 3^{\mathrm{a}}$ harmônica \\
\hline 5999 & $v(\mathrm{CH})^{*}, 1^{\text {a }}$ harmônica & & \\
\hline
\end{tabular}

Onde: $v=$ estiramento, $(\mathrm{CH})^{*}=$ estiramento $\mathrm{CH}$, do anel 
Embora observada no espectro do PMMA, não foi possível identificar no espectro NIR do PC a quinta harmônica de $\mathrm{CO}$, provavelmente, devido à sua baixa intensidade. As bandas observadas na região próxima a $7000 \mathrm{~cm}^{-1}$ devem ser originadas de uma combinação das bandas que ocorrem na região da primeira harmônica com um múltiplo de uma frequiência fundamental, conforme discutido no caso dos outros polímeros. Uma atribuição provável do espectro NIR do PC, baseada nas observações apresentadas, é apresentada na Tabela 13.

\section{Conclusão}

As bandas harmônicas de estiramento $\mathrm{CH}$ para $\Delta \mathrm{v}=2,3 \mathrm{e} 4$, foram observadas em aproximadamente 6000,8500 e $11000 \mathrm{~cm}^{-1}$, no espectro dos polímeros PS, PMMA e PC.

As bandas harmônicas de estiramento $\mathrm{C}=\mathrm{O}$ para $\Delta \mathrm{v}=2,3 \mathrm{e} 4$, foram observadas em 3534, $5264 \mathrm{e}$ $6998 \mathrm{~cm}^{-1}$ no espectro do PC e $\Delta \mathrm{v}=2,3,4$, e 6 em 3437, 5130, 6764 e $10122 \mathrm{~cm}^{-1}$ no espectro do PMMA. A tentativa de atribuição das frequiências dos espectros vibracionais no infravermelho próximo (4000 a $12000 \mathrm{~cm}^{-1}$ ) só foi possível usando-se a aproximação matemática de deconvolução espectral e ajuste de curva, onde se obteve um conjunto de bandas bem definidas, e foi possível efetuar a atribuição de bandas harmônicas de estiramento $\mathrm{CH}$ aos diferentes grupos funcionais $\mathrm{CH}_{2}, \mathrm{CH}_{3}, \mathrm{CH}$ e $\mathrm{CH}$ aromático. Para o PMMA, entretanto, não foi possível identificar as bandas harmônicas que pudessem ser atribuídas aos diferentes grupos funcionais $\mathrm{CH}_{2}$, $\mathrm{CCH}_{3}$ e $\mathrm{OCH}_{3}$.

As correções de anarmonicidade calculadas nos gráficos de Birge - Sponer apresentaram valores para o estiramento $\mathrm{CH}$, e $\mathrm{CO}$, tipicamente da ordem de 59 e $10 \mathrm{~cm}^{-1}$, respectivamente.

Os espectros no NIR desses polímeros podem ser interpretados satisfatoriamente usando a aproximação de modos locais, que foi desenvolvida para interpretar o espectro no NIR de moléculas orgânicas.

\section{Agradecimentos}

FAPESP, $\mathrm{CNP}_{\mathrm{q}}$.

\section{Referência Bibliográfica}

1. Lachenal, G. Analusis Magazine, 26 (4), p. M20 - M29 (1998).
2. Hognigs, D. E.; Hirshfeld, T. B.; Hiefteje, G. M. Anal. Chem. 57 (2), p. 443 - 445 (1985).

3. Miller, C. E.; Edelman, P. G. \& Ratner, B. D. App. Spectrosc. 44 (4), p. 576 - 580 (1990).

4. Davies, A. M. C.; Grant, A.; Gavrel, G. M. \& Steeper, V. Analyst, 110, p. 643 - 647 (1985).

5. Miller, C. E. \& Eichinger, B. E. App. Spectrosc. 44, (3), p. $496-504$ (1990).

6. Fisher, D. J. \& Eichhorn, K.-J. Analusis Magazine, 26 (4), p. M58 - M64 (1998).

7. Shimoyama, M.; Hayano, S.; Matsukawa, K.; Inoue, H.; Ninomiya. T. \& Ozaki, Y. J. Polym. Sci.: Part B: Polym. Phys. 36, p. 1529 - 1537 (1998).

8. Crandall, E. W. J. Chem. Edu. 64, (5), p. 446 - 467 (1987).

9. Takezawa, Y.; Taketani, N.; Tanno, S. \& Ohara, S. J. Polym. Sci.: Part B: Polym. Phys., 30, p. 879 - 885 (1992).

10. Snavely. D. L. \& Dubsky, J. J. Polym. Sci.: Part A: Polym. Chem., 34, p. 2575 - 2579 (1996).

11. Prasad, P. N. \& Williams, D. J. Introduction to Nonlinear Optical Effects in Molecules and Polymers. John Wiley \& Sons, Inc. New York, (1991).

12. Bauer, S. J. Appl. Phys., 80, (10), p. 5533 (1996).

13. McCulloch, I. \& Yoon, H.. J. Polym. Sci.: Part A: Polym. Chem., 33, p. 1177 - 1183 (1995).

14. Beecroft, L. L. \& Ober, C. K.. J. M. S. Pure Appl. Chem. A (34), p. 573 - 586 (1997).

15. Takezawa, Y. \& Ohara, S. J. Appl. Polym. Sci., 49, p. 169 - 173 (1993).

16. Selegue, T. J.; Ranatunga, D. R. A.; Hansosn, K. A. \& Snavely, D. L. Spectroquimica Acta, 46 A, (12), p. 1759 - 1766 (1990).

17. Painter, P. C. \& Koenig, J. K. J. Polym. Sci. Polym. Phys., 15, p. 1885 - 1903 (1977).

18. Mark, H. F.; Bikales, N. M.; Overberger, C. G.; Menges, G.; Kroschwitz, J. I. Encyclopedia of Polymer Science and Engineering, $2^{\mathrm{a}} \mathrm{ED}$, Jonhn Wiley \& Sons, New York, p. 85 (1987)

19. Snavely, D. L. \& Angevine, C. J. Polym. Sci.: Part A: Polym. Chem. 34, p. 1669 - 1673 (1996).

20. Schineider, B.; Stokr, J.; Schidt, P.; Mihailov, M.; Dirchkov, S. \& Peeva, N.. Polymer, 20, p. 705 - 712 (1979).

21. Dirlikov, S. \& Koening, J. Appl. Spectrosc., 33, p. 555-561 (1979).

Recebido:10/08/01

Aprovado: 13/12/01 